\title{
Das Enträtseln der Zelleinschlüsse
}

Ein Beitrag zur Geschichte der Virologie

Peter Diosi

\section{Summary}

In the middle of the $19^{\text {th }}$ century cell inclusions were observed with increasing frequency in more and more diseases and were closely scrutinized by researchers working in different fields. Because of their distinct viewpoints, however, the various authors came inevitably to different conclusions. The morphologists interpreted the inclusions as artefacts or degenerative changes, the etiologists, on the other hand, took them for pathogenic protozoa, for cellular lesions inflicted by invisible agents or, conversely - for aggregated products of the cellular defense. Various morphological, parasitological and bacteriological methods have been used to clear up the pros and cons of these hypotheses. It was the rapid progress realized in virology at the middle of the $20^{\text {th }}$ century that finally brought to light their real significance.

\section{Zusammenfassung}

Als Mitte des 19. Jahrhunderts bei immer mehr Krankheiten immer häufiger Zelleinschlüsse gesichtet wurden, versuchten Forscher verschiedener Fachbereiche diese zu deuten. Weil sie aber die Gebilde aus unterschiedlicher Perspektive betrachteten, kamen sie zwangsläufig zu verschiedenen Auffassungen über deren Natur. Die Morphologen hielten sie für Kunstprodukte oder Degenerationserscheinungen der Zelle, die Ätiologen deuteten sie hingegen als pathogene Protozoen, als von unsichtbaren Erregern verursachte Zellschädigungen, oder umgekehrt - als Reaktionsprodukte der Zelle auf deren Invasion. Im Laufe der Zeit wurden dann verschiedene morphologische, parasitologische und bakteriologische Methoden eingesetzt, um das Für und

Dr. Peter Diosi, Alteburger Straße 298, D-50968 Köln 
Wider dieser Hypothesen zu ergründen. Doch erst Mitte des 20. Jahrhunderts kam durch den atemberaubenden Fortschritt der Virologie ihre echte Natur zum Vorschein.

\section{Einführung}

Als Henderson ${ }^{1}$ und Paterson ${ }^{2} 1841$ bei mehreren Fällen von Molluscum contagiosum im Zytoplasma der Epidermiszellen auf Zelleinschlüsse (ZE) gestossen waren, schien ihnen nichts näherliegend, als diese für den Träger des Ansteckungsstoffes, ja als den Erreger selbst anzusehen. Nachdem aber Karl Weigert ${ }^{3}$ (1845-1904) bei Variola und weitere Forscher auch bei anderen mit charakteristischen Hautveränderungen einhergehenden Krankheiten das Vorkommen ähnlicher Gebilde beobachtet hatten, kamen diesbezüglich Zweifel auf. Mit der Zeit war es durch die Beschreibung allerlei undeutlicher Gebilde als «Einschlüsse» ungemein schwierig, ja fast unmöglich geworden, alle ZE miteinander zu vergleichen, geschweige denn festzustellen, ob es sich jeweils um dieselben Strukturen handelte. Als erstes mussten die in der Literatur zerstreuten Daten zusammengefasst und dann die Natur der ZE über die bequemen Verbalismen «Degeneration», «Kunstprodukt» bzw. «Gerinnungsprodukt» hinaus geklärt werden. Während Gruenhagen ${ }^{4}$ die Gebilde für Blastomyzeten hielt, zählten Otto von Bollinger ${ }^{5}$ (1843-1909), Albert Neisser ${ }^{6}$ (1855-1916) und Ludwig Pfeiffer ${ }^{7}$ (1842-1921) diese den Kokzidien zu. Rudolf Virchow ${ }^{8}$ (1821-1902) und Paul Unna ${ }^{9}$

1 William Henderson (Edinburgh), «Notice of the Molluscum Contagiosum.» Edinburgh med. surg. J., 56/148 (July 1, 1841) 213-218.

2 Robert Paterson (Leith), "Cases and Observations on the Molluscum Contagiosum of Bateman, with an Account on the Minute Structure of the Tumors.» Edinburgh med. surg. J., 56/148 (July 1, 1841) 279-288.

3 Carl Weigert (Breslau), «Anatomische Beiträge zur Lehre von den Pocken. 1. Heft: Die Pocken-Effloreszenz der äusseren Haut. II. Theil: Ueber pockenähnliche Gebilde in parenchymatösen Organen und deren Beziehungen zu Bakteriencolonien.» Max Cohn \& Carl Weigert Vlg., Breslau, 1874/1875, 41.

4 A. Gruenhagen (Königsberg), «Bemerkungen über den Infektionsstoff der Lymphe.» Arch. Dermat. Syph. (Berlin), 4 (1872) 150-152.

5 Otto von Bollinger (München), «Über Epithelioma contagiosum beim Haushuhn und die sog. Pocken des Geflügels.» Virchows Arch. path. Anat. (Berlin), 58/4 (1873) 349-356.

6 Albert L. S. Neisser (Leipzig), «Über die parasitäre Natur des Molluscum contagiosum.» Monatshefte prakt. Dermatol. (Hamburg/Leipzig), 1/1 (März 1882) 17-19.

7 Ludwig Pfeiffer (Weimar), «Ein neuer Parasit der Pockenprozesse aus der Classe Sporozoa (Leuckhart).» Monatshft. prakt. Dermatol. (Hamburg/Leipzig), 6/10 (15. Mai 1887) 435-447.

8 Rudolf Virchow (Berlin), «Über Molluscum contagiosum.»Virchows Arch. path. Anat. (Berlin), 33/1 (Mai 1865) 144-154.

9 Paul Gerson Unna (Hamburg), «Histopathologie der Hautkrankheiten.» Leopold Voss Vlg., Leipzig, 1894. 
(1850-1929) betrachteten die ZE hingegen als eine besondere Art von Degeneration epidermaler Elemente. Später wurden morphologische, parasitologische und bakteriologische Methoden eingesetzt, um das Für und Wider dieser Hypothesen zu ergründen.

Auguste Chauveau ${ }^{10}$ (Abb. 1, links oben) schlug eine andere Linie ein; er suchte den Vaccineerreger in der Lymphe. Im Jahre 1868 gelang es ihm, experimentell festzustellen, dass der Ansteckungsstoff sich nicht in der serösen Flüssigkeit, sondern in den darin suspendierten kleinsten Körnchen befand. Seine «granulations élémentaires» kündigten die Existenz der Viren voraus.

\section{Kornealtest}

Gailleton ${ }^{11}$ hatte 1889 beobachtet, dass die Kaninchen auf die Verimpfung von Pockenlymphe empfindlich reagierten. Straus, Chambon und Ménard ${ }^{12}$ fügten 1890 hinzu, dass die dünne, avaskuläre Hornhaut der Versuchstiere zur mikroskopischen Untersuchung der Pockenläsionen besser geeignet sei als die zu diesem Zweck bis dahin benutzte vielschichtige und reichlich durchblutete Haut bzw. Mamille.

Giuseppe Guarnieri ${ }^{13}$ (1856-1918) konnte 1892 in der mit Pockenlymphe geimpften Kaninchenhornhaut tatsächlich das Auftreten von bis dahin unbemerkten ZE beobachten. Als in den herdenweise auftretenden pathologischen Veränderungen der Hornhaut dieselben Gebilde zum Vorschein traten, die ihm in der Haut von Pockenkranken begegnet waren, begriff er sofort, dass es ihm gelungen war, die Pockenerreger zu züchten.

\section{Protozoenhypothese}

Bei 500facher Vergrösserung hatte Guarnieri im Zytoplasma der erkrankten Epidermiszellen runde bis ovale, scharf umrissene und stets von hellem Hof umgebene ZE erblickt, die - nach Manns Methode gefärbt - hellrot im

10 Jean Baptiste Auguste Chauveau (Lyon), «Nature du virus vaccin. Détermination expérimentale des éléments qui constituent le principe actif de la sérosité vaccinale virulente.» C. R. Acad. Sci. (Paris), 66 (1868) 289-295.

11 Gailleton, zit. in: Albert Calmette/Camille Guérin (Lille), «Recherches sur la vaccine expérimentale.» Ann. Inst. Pasteur (Paris), 15/1 (Janvier 1901) 161-168, S. 161.

12 Straus/Chambon/Ménard (Paris), «Recherches expérimentales sur la vaccine, chez le veau.» C. R. Soc. Biol. (Paris), Ser. 9 (20 décembre 1890) 721-727.

13 Giuseppe Guarnieri (Pisa), «Ricerche sulla pathogenesi ed etiologia dell'infezione vaccinica e vaiolosa.» Arch. Sci. med. (Torino) 16/22 (1892) 403-424. 
blautingierten Zytoplasma aufleuchteten. Die Körperchen zeigten eine homogene bzw. maulbeerartige Struktur und nahmen zur Peripherie der Herde hin an Grösse erheblich zu.

Guarnieri erschien es überdies, als könne er bei passender Beleuchtung mit einem starken Linsensystem Kern und Protoplasma in den ZE unterscheiden und Lebenserscheinungen wie amöboide Bewegungen und binäre Teilung beobachten. Er zweifelte daher nicht weiter daran, dass er auf den viel gesuchten Pockenerreger gestossen war, und glaubte, in den Gebilden Protozoen, vermeintlich Rhizopoden zu erblicken.

\section{Cytoryctes variolae}

Der helle Hof um die ZE erweckte in Guarnieri den Eindruck, als habe der Schmarotzer das Protoplasma um sich her aufgezehrt, und er bezeichnete diesen daher mit dem griechischen Wort für «Verzehren» als Cytoryctes vaccinae bzw. variolae. Andere Forscher hatten den Halo um die Guarnierischen Körperchen jedoch als Phagozytose-Vakuole bzw. als Kunstprodukt ${ }^{14}$ ausgelegt.

Aus Guarnieris Beobachtungen war die Art und Weise, wie sich der vermutete Parasit gebildet und vermehrt haben sollte, nicht lückenlos nachvollziehbar. Fest steht, dass er die unterschiedliche Grösse der Körperchen als Zeichen von Wachstum bzw. als deren Entwicklungsstadien ausgelegt hatte. Erst Jahre später unternahm Calkins ${ }^{15}$ den ebenso kühnen wie mühsamen Versuch, aus den spärlichen und in ihrer Deutung zweifelhaften Beobachtungen den vollständigen Lebenszyklus des Cytoryctes zu rekonstruieren.

\section{Die Streitfrage der Spezifität}

Während sich Guarnieri auf die Beobachtung eventueller Lebenserscheinungen des Parasiten konzentriert hatte, beschäftigte die Nachuntersucher vielmehr die Frage, ob die Guarnierischen Körperchen pockenspezifische Erscheinungen seien. Ihre Nachprüfungen zeigten, dass die Gebilde bei Pocken konstant, in anderen pathologischen Prozessen und in gesunden Ge-

14 O. Casagrandi (Cagliari), «L'Etiologia del vaiuolo umano.» Ann. Ig. Speriment., 20/1 (1910) 39-44.

15 Gary Nathan Calkins (New York), «The Life History of Cytoryctes Variolae, Guarnieri.» J. med. Res. (Boston), 11 (February 1904) 136-172. 

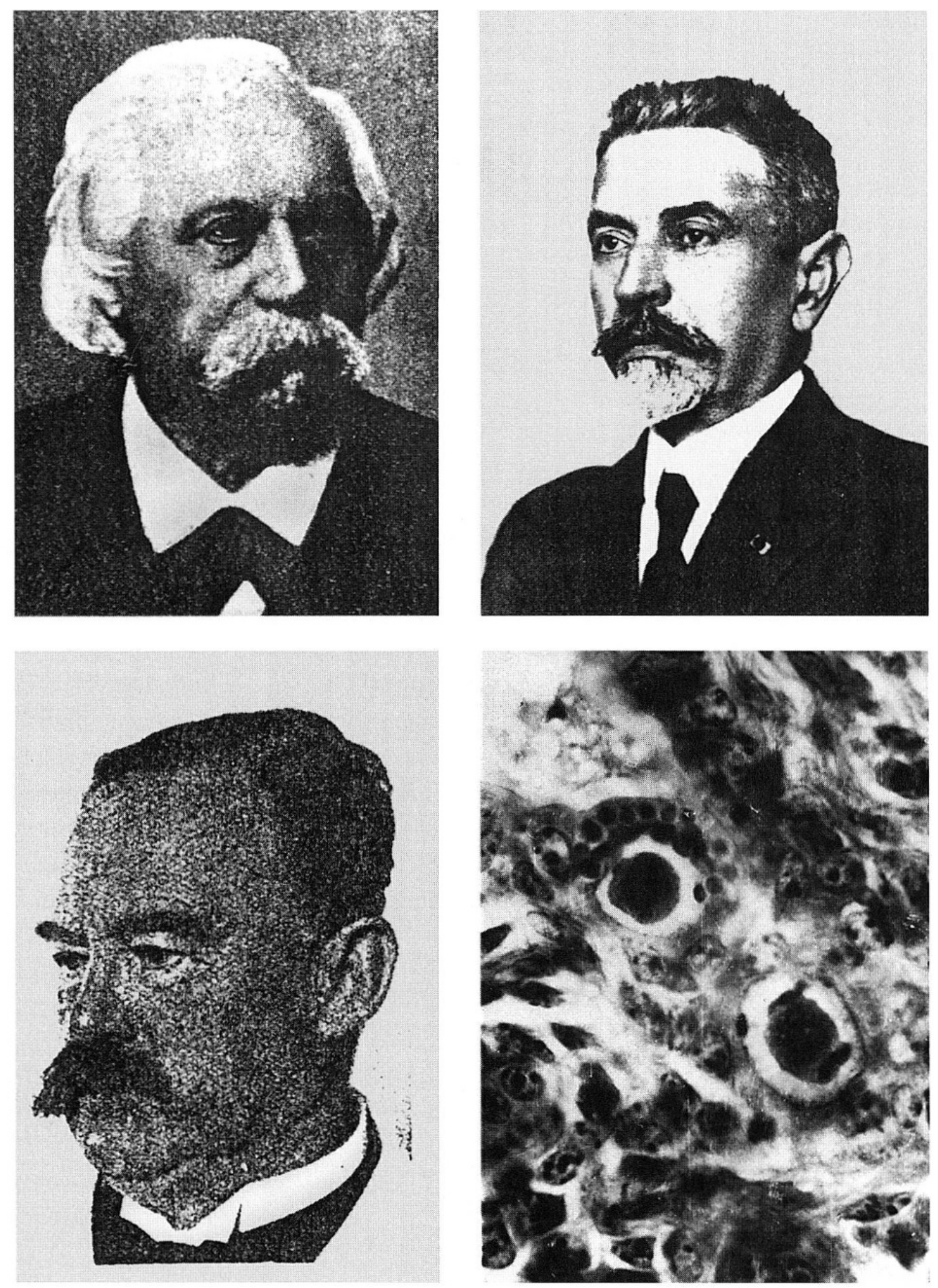

Abb. 1. Jean Baptiste Auguste Chauveau (1827-1917) (oben, links); Amédé Borrel (1867-1936) (oben, rechts); Albert Jesionek (1870-1935) (unten, links); Zytomegalie der Speicheldrüse beim Meerschweinchen. Zwei vergrösserte, jeweils von einem Halo umgebene Kernkörper und mehrere kleine Plasmaeinschlüsse aufweisende Epithelzellen. (Mikrophotographie, aus der Kollektion des Verfassers; unten, rechts.) 
weben aber niemals auftraten. Theodor von Wasielewski ${ }^{16}$ (1868-1941) konnte die spezifische Natur der Guarnierischen Körperchen 1901 auch experimentell nachweisen, indem er die Gebilde in der Kaninchenhornhaut nach dem Auftragen von Pockenlymphe ständig, sonst aber nie auffand. Andere Forscher ${ }^{17,18}$ bestätigten seine Beweisführung indirekt dadurch, dass es keinem von ihnen gelungen war, ähnliche Veränderungen bei mechanischer und chemischer Reizung oder nach Verimpfung anderer Erreger überzeugend nachzuweisen.

Die Skeptiker warnten jedoch vor voreiligen Schlussfolgerungen, da ähnliche Gebilde auch bei anderen Krankheiten und gelegentlich sogar bei Gesunden beobachtet worden waren.

\section{Polarisierung der Auffassungen}

Während die an ätiologischer Forschung interessierten Wissenschaftler (kurz Ätiologen) die Protozoennatur der Guarnierikörperchen für so gut wie erwiesen hielten und nunmehr die Biologie und Entwicklungsgeschichte des Parasiten zu erörtern versuchten, standen die Morphologen dieser Auffassung ablehnend gegenüber. Sie hielten die Ätiologen - schon wegen deren Zielsetzung, nach Krankheitserregern zu fahnden - für unfähig, mit ungelösten Problemen unbefangen umzugehen. Sie selber sahen im Suchen nach fragwürdigen Erregern keine lohnende Arbeit und bemühten sich vielmehr, die Phänomene unvoreingenommen auf rein morphologischer Basis wahrzunehmen. In ihren Augen waren die als Protozoen ausgelegten Gebilde Degenerationsprodukte des Gewebes, ausgestossenes Kernmaterial oder Reste einverleibter Wanderzellen.

Die Ätiologen hatten ihre Widersacher daraufhin, mit Anspielung auf deren knappe empirische Denkweise, als «genaue Nurbeobachter» verspottet. Sie betrachteten die ZE ihrerseits als ein ungleich komplizierteres Problem, das nur vom Ganzen her unter Berücksichtigung von experimentellen Daten und biologischen Kriterien entsprechend beurteilt werden konnte.

16 Theodor von Wasielewski (Halle/Saale), «Beiträge zur Kenntnis des Vaccine-Erregers.» Zschr. Hyg. Infektionskr. (Leipzig), 38 (1901) 212-318.

17 Paul Salmon (Paris), «Recherches sur l'infection dans la vaccine et la variole.» Ann. Inst. Pasteur (Paris), 11/4 (25 Avril 1897) 289-307.

18 C. Gorini (Roma), «Über die bei der Vaccine ausgeführten Hornhautimpfung vorkommenden Zelleinschlüsse und über deren Beziehung zu Zellinklusionen der bösartigen Geschwülste.Vorläufige Mitteilung.»Cbl.Bakt.I.Abt. Orig.A (Jena),28/8-9 (4.September 1900) 233-237. 


\section{Ausgestossene Nukleolen? ...}

Victor Babes ${ }^{19}$ (1854-1926) hatte 1893 auf dem 11. Ärztekongress zu Rom grosses Aufsehen mit der Behauptung erregt, dass es sich bei den ZE möglicherweise, ja wahrscheinlich um aus dem Zellkern ausgewanderte Nukleolen handelte. Babes war von der Beobachtung ausgegangen, dass aus dem Kern der ZE-aufweisenden Zellen die Nukleolen häufig fehlten, und folgerte mit bestechender Argumentation, dass diese eigentlich aus dem Zellkern ausgestossene entartete Nukleolen seien - eine Ansicht, der auch Sanfelice ${ }^{20}$ zustimmte.

Wasielewski ${ }^{21}$ schloss die Abstammung der ZE von Nukleolen schon deshalb aus, weil letztere unter Anwendung des von Gustav Giemsa ${ }^{22}$ (1867-1948) entwickelten Färbeverfahrens immer noch im Zellkern nachweisbar waren. Laut Hücke ${ }^{23}$ konnte von der Ableitung der ZE vom übrigen Kernmaterial auch keine Rede sein, da sich die Kerne der befallenen Zellen normal verhielten und ungehindert weiter teilen konnten. Hückel erinnerte ferner daran, dass in den nach Giemsa gefärbten Präparaten die Nukleolen stets tiefblau, die ZE hingegen schön rot erschienen und dadurch leicht voneinander unterschieden werden konnten. Bosc ${ }^{24}$ wies schliesslich darauf hin, dass der von Babes angesprochene Umwandlungsprozess der Nukleolen in Wirklichkeit niemals beobachtet wurde.

\section{... oder einverleibte Blutkörperchen?}

Salmon $^{25}$ aus Metschnikoffs Laboratorium hielt die ZE eher für Reste einverleibter Erythro- bzw. Leukozyten. Auch Ferroni und Massari ${ }^{26}$ teilten diese Ansicht.

19 Victor Babes (Paris), «Discussion» (im Anschluss an Guarnieris Vortrag). Atti dell'XI. Congresso medico internazionale (Roma), 2 (1894) 134.

20 Francesco Sanfelice (Modena), «Recherches sur la genèse des corpuscules du Molluscum contagiosum (Mémoire publié à l'occasion du Jubilé de E. Metchnikoff)», Ann. Inst. Pasteur (Paris), 32/8 (Août 1918) 363-373, hier 369.

21 Wasielewski (oben Anm. 16).

22 Gustav Giemsa (Hamburg), «Geschichte, Theorie und Weiterentwicklung der RomanowskyFärbung.» Med. Welt. (Berlin/Stuttgart), 8/41 (13. Oktober 1934) 1432-1434.

23 Armand Hückel (Tübingen), «Die Vaccinekörperchen. Nach Untersuchungen an der geimpften Hornhaut des Kaninchens. Ein experimenteller und kritischer Beitrag zur Frage nach dem Contagium der Vaccine.» Beitr. path. Anat. allg. Path. (Jena), Suppl. 2 (1898) 1-130.

24 F.-J. Bosc (Montpellier), «Les epitheliomas parasitaires. La clavelée et l'epithelioma claveleux.» Cbl. Bakt. I. Abt. Orig. A (Jena), 34/5 (Mai 1903) 413-420; 34/6 (Juni 1903) 517-526; 34/7 (Juli 1903) 666-674, S. 667.

25 Salmon (oben Anm. 17).

26 E. Ferroni/G. Massari (Catania), «Sulla pretesa scoperta del Guarnieri riguardo la infezione vaccinica e vaiolosa. Nota preliminare.» Riforma med. (Napoli), 9/126 (1893) 602-604, hier 603. 
Die Anhänger der Protozoentheorie versuchten auch diese Hypothese mit einer Vielzahl von Argumenten zu widerlegen. Sie bezweifelten, dass in der avaskulären Hornhaut ausgebluteter Kaninchen Erythrozyten überhaupt vorkommen könnten, und betonten, dass im Vergleich zu der enormen Zahl der ZE in den Pockenläsionen die Zahl der eingewanderten Leukozyten unerheblich sei und ihre Einwanderung ohnehin durch Opiumbepinselung verhindert werden konnte. Wasielewski betonte ferner, dass die Leukozyten anhand ihrer eigentümlichen Tuschaufnahme und Farbaffinität leicht als solche identifizierbar waren. Während nämlich die Kernreste von Leukozyten sich mit Alaunfuchsin-Hämatoxylin tiefblau, mit Jodgrünfuchsin violett und mit Biondis Mischung grün färbten, nahmen die ZE eine rote, hellrote bzw. violette Farbe an.

Stanislaus von Prowazek ${ }^{27}$ war es letztendlich mit der Verimpfung der Pockenlymphe in vitro auf die ausgeschnittene Kaninchenhornhaut gelungen, den Verdacht auf Verwechslung mit eingewanderten Leukozyten endgültig zu zerstreuen.

\section{Heftige Auseinandersetzungen}

Die Morphologen betrachteten die besonders von William T. Councilman ${ }^{28}$ (1859-1933) aufrecht erhaltene Protozoenhypothese als absurd. Ihr Hauptvorwurf galt dem Fehlen aller Kennzeichen der Protozoen: die ZE zeigten weder Kerne noch Zysten noch Entwicklungsstadien. Die von Guarnieri als «Kerne» ausgelegten Körnchen hatten sich ja als Chromatinbrocken ${ }^{29}$ und die auf binäre Teilung hinweisenden Zeichen als durch Flachschnitte vorgetäuschte Bilder herausgestellt. Hückel ${ }^{30}$ sprach daher in aller Deutlichkeit aus, dass die ZE gar nichts mit Protozoen zu tun haben konnten und das Fehlen von Entwicklungsstufen entschieden gegen deren Einreihung unter Rhizopoden spräche. Selbst denjenigen Morphologen, welche die pockenspezifische Natur der Guarnierikörperchen nicht unbedingt ablehnten,

27 Stanislaus J. M. von Prowazek (Hamburg), «Vaccine.» In: Stanislaus von Prowazek (Hrsg.), Handbuch der pathogenen Protozoen. Johann Ambrosius Barth Vlg., Leipzig, 1912, Bd. I, 122-138.

28 William Thomas Councilman/George Burgess Magrath/Walter Remsen Brinkerhoff (Boston, Mass.), «The Pathological Anatomy and Histology of Variola.» J. med. Res. (Boston), 11 (February 1904) 12-135.

29 Gorini (oben Anm. 18).

30 Hückel (oben Anm. 23). 
schien die Schlussfolgerung, dass diese auch nur eventuell als die Erreger der Pocken anzusehen wären, durchaus unberechtigt.

Die Anhänger der Protozoenhypothese hatten hingegen die Auffassung der Morphologen, wonach die ZE Degenerationsprodukte der Zelle wären, als eine willkürliche, durch nichts unterstützte Hypothese betrachtet. Sie unterstellten ihren Widersachern, dass diese sich vor der Verantwortung drückten und auf der Degenerationshypothese nur deshalb beharrten, weil sie nicht ätiologischer Natur war. Sie warfen ihren Gegnern vor, dass obwohl die Medizin sich durchweg mit Indizienbeweisen begnügen musste diese seitens der ätiologischen Forschung auf eindeutigen Beweisen bestanden und ihr Fehlen als Argument für die degenerative Natur der ZE geltend machten. Henrique da Rocha-Lima ${ }^{31}$ (1879-1920) beschuldigte sie, dass ihr Misstrauen zu weit ging und dass sie oft selbst sorgfältig beobachteten und kritisch gedeuteten Befunden weniger Glauben schenkten als einer reinen Hypothese aus einem anderen Gebiet.

\section{Woher stammten die Gegensätze?}

Nach René DuCastels ${ }^{32}$ (1845-1905) Ansicht hatte sich jede Generation eine der gerade herrschenden Anschauungen entsprechende Idee zurechtgelegt, und die Gegensätze konnten demnach auf den Generationenkonflikt zurückgeführt werden. Burnet ${ }^{33}$ schrieb die Meinungsverschiedenheiten eher der Tatsache zu, dass die anfangs als organisierte Strukturen imponierenden $\mathrm{ZE}$ später zu amorphen Brocken zerfielen und nach dem Zeitpunkt der Beobachtung unterschiedlich ausgelegt werden konnten. Die zur psychologischen Betrachtung neigenden Kritiker leiteten die Gegensätze hingegen aus der unterschiedlichen Mentalität und seelischen Struktur der Forscher ab. DaRocha-Lima ${ }^{34}$ schrieb sie schliesslich dem Versagen, die Erregernatur der ZE im Sinne Kochs zu beweisen, zu.

31 Henrique da Rocha-Lima (Hamburg), «Zur Demonstration über Chlamydozoen.»Verh. dtsch. path. Ges. (Berlin), 16 (1913) 198-210, hier 199.

32 Auguste Marie René Du Castel, zit. in: Benjamin Lipschütz (Wien), «Untersuchungen über die Ätiologie der Krankheiten der Herpesgruppe (Herpes zoster, Herpes genitalis, Herpes febrilis).» Arch. Dermat. Syph. (Berlin), 136/3 (2. Dezember 1921) 428-482, hier 430.

33 Etienne Burnet (Paris), «Contribution à l'étude de l'Epithélioma contagieux des oiseaux.» Ann. Inst. Pasteur (Paris), 20/9 (septembre 1906) 742-765, hier 758.

34 DaRocha-Lima (oben Anm. 31), 199. 
Wie dem auch sei, die Heftigkeit der Auseinandersetzungen deutete darauf hin, dass die Erforschung der ZE sich festgefahren hatte. Neue Leute, frische Ideen und verfeinerte technische Verfahren waren gefragt, um den Weg aus der Sackgasse zu finden.

\section{Sprung zum Unsichtbaren}

Der Begriff «invisible Viren» rührte von Louis Pasteur (1822-1895) her. Nach jahrelanger erfolgloser Bemühung, den Erreger der Tollwut zu Gesicht zu bekommen, soll er sich 1881 dahin geäussert haben, dass das Lyssavirus unsichtbar klein sein dürfte ${ }^{35}$. Um so grösser war die Überraschung, als Negri ${ }^{36} 1903$ im Gehirn tollwutkranker Tiere auffällige ZE nachweisen konnte, welche während der langen Suche nach dem Lyssavirus einer Reihe namhafter Wissenschaftler entgangen waren. Ilja Metschnikoff (1845-1916) betrachtete auch den Pockenerreger als ein winziges pflanzliches Wesen, das seiner Kleinheit wegen für immer unsichtbar bleiben werde. Émile Roux ${ }^{37}$ (1853-1933) folgerte hieraus, dass die Viren im allgemeinen virtuelle Wesen, «être de raison», seien.

Eine exakte experimentelle Begründung erfuhr diese Vorstellung 1890, als Dmitri Iwanowski ${ }^{38}$ (1864-1920) das aktive Agens der Mosaikkrankheit im Filtrat kranker Tabakblätter gefunden hatte. Nachdem es dann Friedrich J. A. Löffler (1852-1915) und Paul Frosch ${ }^{39}$ (1860-1928) bei der Maul- und Klauenseuche gelungen war, die Infektiosität des Filtrates der Blasenflüssigkeit durch fortlaufende Verdünnungen zu titrieren, schwenkte das Interesse der Ätiologen von den ZE plötzlich auf das Filtrat der pathologischen Absonderungen. Die von Tiegel ${ }^{40} 1871$ ursprünglich zur Zurückhaltung von Bakterien eingeführten Kieselgurfilter wurden kurzerhand zur Gewinnung von Filtrat verwendet.

35 Émile Roux (Paris), «Sur les microbes dits 〈invisibles».» Bull. Inst. Pasteur (Paris), 1/1 (28 février 1903) 7-12.

36 Adelchi Negri (Roma), «Beitrag zum Studium der Etiologie der Tollwuth.» Zschr. Hyg. Infektionskr. (Leipzig), 44 (1903) 519-540.

37 Roux (oben Anm. 35).

38 Dmitri Jossifowitsch Iwanowski (St. Petersburg), «Die Mosaikkrankheit der Tabakpflanze.» (Russisch), Bull. Acad. Sci. Imper. (St. Petersburg), Serie T, $37 / 7$ (1890) 239.

39 Friedrich A. J. Loeffler/Paul Frosch (Berlin), «Berichte der Komission zur Erforschung der Maul- und Klauenseuche bei dem Institut für Infektionskrankheiten in Berlin. Erstattet an den Kultusminister.» Cbl. Bakt. I. Abt. Orig A. (Jena), 23/8 (27. Februar 1898) 371-391.

40 E. Tiegel (Schaffhausen), «Ursache des Milzbrandes.» Korrespbl. Schweiz. Ärzte (Bern), 1/10 (1. Oktober 1871) 275-280. 


\section{Filtrierbare Viren}

Adelchi Negri ${ }^{41}$ (1876-1912) führte die anfänglichen Misserfolge der Übertragungsversuche des Pocken-Erregers mittels Filtrat auf den Umstand zurück, dass die Viren innig mit der Zelle verbunden waren. Er hatte erkannt, dass das pathologische Material vor dem Filtrieren gelockert und verdünnt werden musste, um den eng mit dem Zelldetritus verbundenen Erreger für das Filtrieren freizustellen. Nach wenigen fehlgeschlagenen Versuchen war es mit seiner Methode auch bei anderen Krankheiten gelungen, die Virulenz des Filtrates zu bestätigen.

Mit dem Nachweis der Filtrierbarkeit des Infektionsstoffes hatten sämtliche Protozoenbefunde jede Bedeutung verloren. Es war das Ende einer Epoche, doch Negri war sich dessen nicht bewusst. Er blieb der Ansicht, dass die filtrierbare Form nur eine Entwicklungsphase des Parasiten darstellte, der später innerhalb der Epithelzellen zu grösseren, die Filter nicht passierenden Formen heranwuchs. Doch Prowaze ${ }^{42}$ gelang es mit der gleichermassen wirksamen Verimpfung von 8- bis 336stündigem Korneamaterial zu beweisen, dass die Filtrierbarkeit des Erregers von den vermuteten Entwicklungsphasen unabhängig ist. Es blieb nur zu klären, ob es sich beim filtrierbaren Infektionsstoff um ein lösliches Toxin oder einen überaus kleinen, die bakteriendichten Filter passierenden Mikroorganismus handelte.

\section{Die Toxinhypothese}

Hücke ${ }^{43}$ hielt die Guarnierischen Körperchen für das Produkt eines durch ein hypothetisches Pockengift ausgelösten Degenerationsprozesses der Zelle, der durch die Trennung der erythrophilen und zyanophilen Komponenten des Protoplasmas zur Erscheinung von ZE führte. Auch Sanfelice ${ }^{44}$ schrieb das Entstehen der ZE der Wirkung eines organischen Toxins zu, welches sich in Kontakt mit der Zelle neu bilden könnte.

Die Ätiologen schlossen sich Jakob Henles ${ }^{45}$ (1809-1885) Auffassung an, wonach eine fortlaufende Übertragung nur möglich sei, sofern sich das

41 Adelchi Negri (Roma), «Esperienze sulla filtrazione del virus vaccinico. Nota prima.» Gazz. med. ital. (Torino), 54/13 (30. marzo 1905) 305-311.

42 Prowazek (oben Anm. 27).

43 Hückel (oben Anm. 23).

44 Sanfelice (oben Anm. 20).

45 Friedrich Gustav Jakob Henle (Göttingen), «Von den Miasmen und Kontagien und von den miasmatisch-kontagiösen Krankheiten.» Klassiker der Medizin, Bd. III, Johann Ambrosius Barth Vlg., Leipzig, 1910. 
pathogene Agens im kranken Organismus wie ein lebendiger Parasit vermehrt. Wasielewski ${ }^{46}$ hielt die Toxinhypothese auch deshalb für unwahrscheinlich, weil die befallenen Zellen keine Degenerationserscheinungen aufwiesen und das Filtrat der Pockenlymphe keine Wirkung auf die normale Zellteilung auszuüben schien. Andere Forscher waren aufgrund ihrer fehlgeschlagenen Übertragungsversuche ebenfalls zu dem Schluss gelangt, dass der Erreger keine chemische Substanz, sondern vermutlich ein unsichtbar kleines, sich durch vitale Aktivität vermehrendes Wesen sei.

Es war aber schwierig, ein filtrierbares Lebewesen sich gestaltlich vorzustellen. Beijerinck ${ }^{47}$ betrachtete das in Gelatine gleichmässig diffundierende Tabakmosaikvirus als ein «lebend-flüssiges» Prinzip, Contagium vivum fluidum. Die Zeitgenossen hielten diese Vorstellung wegen des inneren Widerspruchs zwischen «lebend» und «flüssig» für absurd und muteten den filtrierbaren Viren eher eine spiralig gewundene Struktur zu. Später wurde Beijerinck jedoch aufgrund der intuitiven Abgrenzung des Tabakmosaikvirus von Toxinen und Mikroben als Begründer der Virologie betrachtet ${ }^{48}$.

\section{Korpuskuläre Beschaffenheit der Viren}

Während Beijerinck 1898 seine Untersuchungen publizierte, war es Edmond Nocard (1850-1903) und Émile Roux ${ }^{49}$ gelungen, nachzuweisen, dass die Peripneumonie der Rinder durch eine auf Serumpeptonagar züchtbare und mikroskopisch sichtbare Mikrobe hervorgerufen wird, welche die Chamberland $\mathrm{F}$ und Berkefeld Filter passierte. Dieser Befund wies darauf hin, dass die filtrierbaren Erreger nicht unbedingt unsichtbar, aber durchaus korpuskulärer Natur waren.

Prowazek und Araga ${ }^{50}$ liessen sich zum Nachweis der Körperhaftigkeit des Pockenerregers 1908 ein geistreiches Experiment einfallen. Sie versuchten, die Viren aus dem aktiven Filtrat der Pockenlymphe mit Hilfe der von Bechhold ${ }^{51} 1907$ eingeführten Kolloidfilter (Ultrafilter) anzureichern

46 Wasielewski (oben Anm. 16).

47 M. W. Beijerinck (Amsterdam), «Über ein Contagium vivum fluidum als Ursache der Fleckenkrankheit der Tabakblätter.» Verh. Akad. Wet. Amst. (Amsterdam), 6/5 (1898).

48 André Lwoff (Paris), «The Concept of Virus. The Third Marjory Stephenson Memorial Lecture.» J. gen. Microbiol. (Cambridge), 1/17 (23. August 1957) 239-253.

49 Edmond Isidore Etienne Nocard/Émile Roux (Paris), «Le microbe de la peripneumonie.» Ann. Inst. Pasteur Paris, 12 (1898) 240-247.

50 Stanislaus J. M. von Prowazek (Hamburg)/H. de Beauepaire Aragaõ (Mañguinhos / Rio de Janeiro), «Untersuchungen über Variola.» Münch. med. Wschr., 55/44 (3. November 1908) 2265-2266.

51 H. Bechhold (Frankfurt), «Kolloidstudien mit der Filtrationsmethode.» Zschr. Physik. Chem. (Leipzig), 60/3 (6. August 1907) 257-318. 
und zu isolieren. Da der Durchmesser der Viruspartikel die Porengrösse der Kolloidschicht noch um einiges übertraf, wurden die Viren im Belag zurückgehalten und konnten nach Giemsa gefärbt als winzige rote Körnchen dargestellt werden. Mit diesem Versuch hatte Prowazek die Aufmerksamkeit vom Filtrat erneut auf den Belag gelenkt.

\section{An der Grenze der Sichtbarkeit}

Es verbreitete sich allmählich der Gedanke einer ununterbrochenen Grössenskala der Krankheitserreger. Nach der Berechnung Ernst Abbes ${ }^{52}$ (1840-1905) lagen fünf- bis zehnfach kleinere Körnchen als die kleinsten bekannten Mikroben schon unter der lichtmikroskopischen Wahrnehmungsschwelle. Amédé Borre ${ }^{53}$ (Abb. 1, rechts oben) griff daher zur Darstellung der vermutlich noch vielfach kleineren filtrierbaren Viren auf Löfflers Fuchsintinte zurück.

Friedrich Löffler ${ }^{54}$ (1852-1915) hatte diese Mischung von Beize und Farbstoff 1889 zur Geisselfärbung entwickelt. Die Beize bewirkte die Ablagerung einer dicken Farbschicht auf die zarten Geisselfäden, wodurch diese unter Ölimmersion deutlich in Erscheinung traten. Mit Hilfe dieses Verfahrens gelang es Borrel, bei Vogelpocken innerhalb der lädierten Zellen unzählige etwa $1 / 4 \mu$ grosse leuchtend rote Körnchen nachzuweisen, welche bei der Anwendung herkömmlicher Färbeverfahren der Beobachtung offenbar entgangen waren.

\section{Die Elementarkörper}

Borre ${ }^{55}$ hatte aufgrund seines Befundes gleich ein Modell der filtrierbaren Viren entworfen. Er definierte diese als auf niedrigster Entwicklungsstufe befindliche und von einer schleimartigen Hülle («enveloppe muqueuse») umgebene kleinste Kokken, sog. «corpuscules élémentaires», welche sich im Gegensatz zu den Bakterien - innerhalb der Zelle vermehrten.

52 Ernst Abbé (Jena), «Beiträge zur Theorie des Mikroskops und der mikroskopischen Wahrnehmung.» Arch. Mikrosk. Anat. Entwicklgesch. (Bonn), 9 (1873) 413-419.

53 Amédé Borrel (Paris), «Sur les inclusions de l'épithelioma contagieux des oiseaux (Molluscum contagiosum).»C. R. Soc. Biol. (Paris), 2 (24 décembre 1904) 642-643.

54 Friedrich A.J.Löffler (Greifswald), «Eine neue Methode zum Färben der Mikroorganismen, im besonderen ihrer Wimperhaare und Geißeln.» Cbl. Bakt. I. Abt. Orig. A (Jena), 6/8-9 (18. August 1889) 209-224.

55 Amédé Borrel (Paris), «Microbes dits invisibles et surcoloration.» C. R. Soc. Biol. (Paris), 61/2 (18 Décembre 1909) 774-775. 
Prowazek ${ }^{56}$ stimmte dem sofort zu. Er hatte ja in den Guarnierikörperchen 1903 selber kleinste, an der Grenze der Sichtbarkeit stehende Körnchen erblickt, welche eventuell die Pockenerreger verkörperten, die er aber zunächst nicht mit Sicherheit von Degenerationselementen der Zelle unterscheiden konnte. MacCallum und Oppenheimer ${ }^{57}$ gelang es später, die winzigen Körnchen von der Pockenlymphe durch Zentrifugalkraft zu trennen und zu beweisen, dass das Virus wesentlich mit den Körnchen verbunden war und in der flüssigen Phase vollständig fehlte.

\section{Assoziierung der Viren mit den Zelleinschlïssen}

Borre ${ }^{58}$ beschäftigte unterdessen die Frage, ob zwischen den ZE und Viren irgendein Zusammenhang bestand.

Als er in den nach Giemsa gefärbten Gewebesektionen stets auf ZE, in den aus demselben Material gefertigten und nach Löffler gefärbten Ausstrichpräparaten hingegen jeweils auf einen Haufen von Elementarkörpern gestossen war, schien es ihm unmöglich festzustellen, ob beide Gebilde in derselben Zelle bzw. in Verbindung miteinander auftraten.

Burnet $^{59}$ vermutete, dass es zwischen den Viren und der Ausbildung von $Z E$ einen gesetzmässigen Zusammenhang geben müsse. Er nahm an, dass die ZE aus zwei Komponenten, einer von der Zelle gelieferten Grundsubstanz und - in diese eingebettet - einen Haufen kleinster, den Borrelschen Elementarkörpern entsprechenden Körnchen, bestünden. Prowazek ${ }^{60}$ (Abb. 2) gelang es bald, diese Vorstellung beim Trachom zu bestätigen. Er hatte in den Epithelzellen nach Giemsas Verfahren im hellblauen Protoplasma dunkelblau gefärbte ZE beobachtet, in welchen bei genauer Differenzierung eine wachsende Anzahl feiner rotgefärbter Körnchen auftauchte. Er hielt die roten Körnchen für Viruselemente und die blaue amorphe Grundsubstanz für eine Art Hülle (Chlamys), welche als Reaktion der Zelle auf das Eindringen der Viren entstanden war und diese mantelartig umhüllte. Er bezeichnete die Trachomerreger daher - unter Zusammenfügung der grie-

56 Stanislaus J. M.von Prowazek (Hamburg), «Variola.» In:Stanislaus von Prowazek (Hrsg.) Handbuch der pathogenen Protozoen, Johann Ambrosius Barth Vlg., Leipzig, 1912, Bd. I, 139-152.

57 W. G. MacCallum/Elsa Hutzler Oppenheimer (Baltimore), «Differential Centrifugation: A Method for the Study of Filtrable Viruses, as Applied to Vaccinia.» J. am. med. Ass. (Chicago), 78/6 (February 1922) 410-411.

58 Borrel (oben Anm. 53), 643.

59 Burnet (oben Anm. 33).

60 Stanislaus J. M. von Prowazek (Hamburg), «Chlamydozoa. I. Zusammenfassende Übersicht» Arch. Protistenkd. (Jena), 10 (1907) 336-358. 


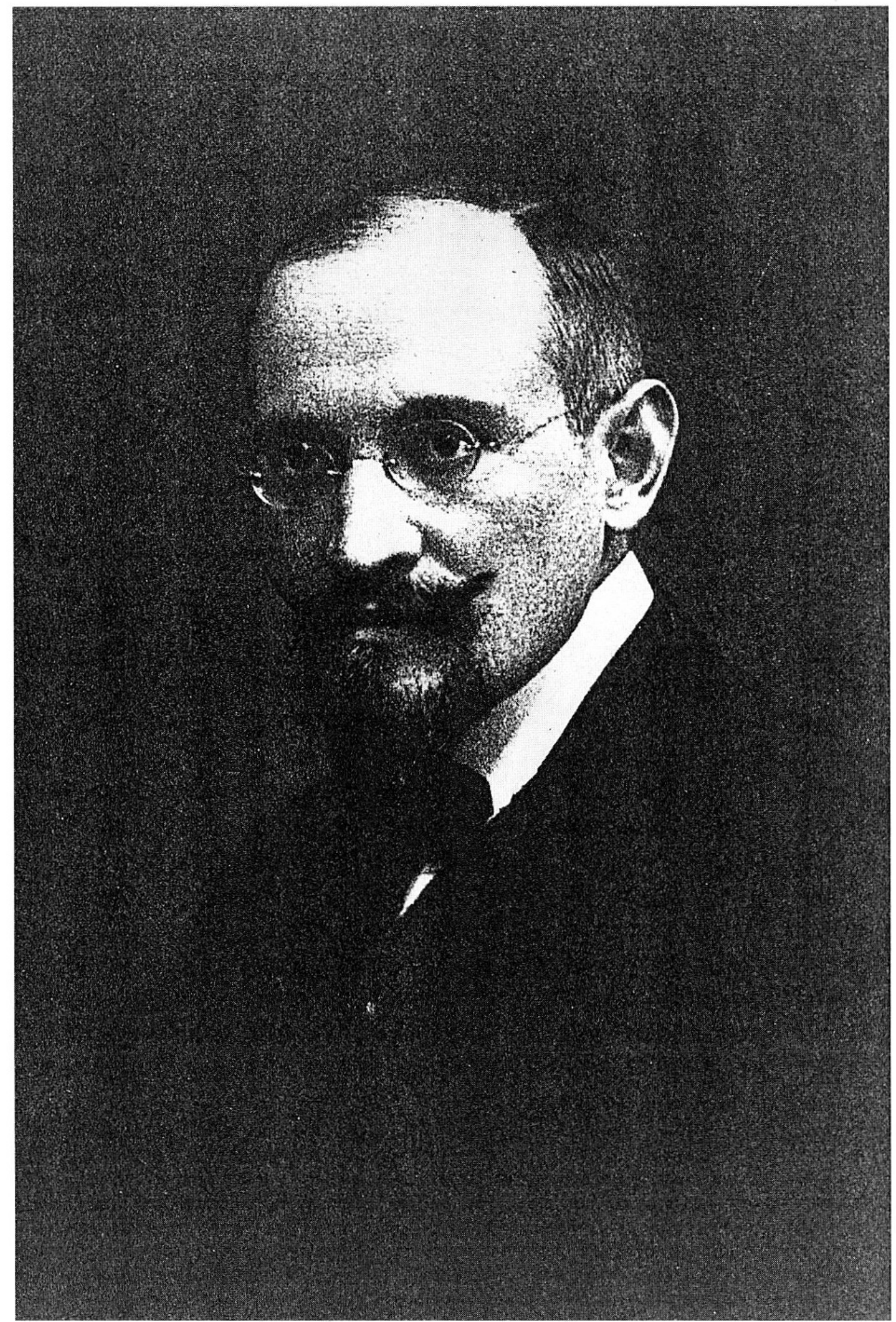

Abb. 2. Stanislaus J. M. von Prowazek (1875-1915). 
chischen Ausdrücke für «Mantel» und «Tiere»- als Chlamydozoa. Die diagnostisch wertvollen ZE kamen seiner Ansicht nach durch diesen Einhüllungsvorgang zustande.

Der Vollständigkeit halber muss jedoch erwähnt werden, dass Lindner ${ }^{61}$ bei den Trachomeinschlüssen keinen «Mantel» im Sinne Prowazeks feststellen konnte und diese als «nackte» Aggregate der Viruselemente betrachtete.

\section{Intrazelluläre Viruskolonien}

Die Chlamydozoen waren Prowazeks ${ }^{62}$ Auffassung nach weder Bakterien noch Protozoen. Sie passierten die gewöhnlichen Bakterienfilter, befielen im kranken Organismus ausschliesslich ektodermale Zellen und konnten sich gleichermassen innerhalb wie ausserhalb der Zelle vermehren. Nach ihrem Eindringen in die Zelle verhielten sie sich anfangs als Symbionten und riefen im Zytoplasma Reaktionsprodukte, die ZE, hervor, um sich dann dem Parasitismus hinzugeben und die Wirtszelle zu zerstören.

Ernest W. Goodpasture ${ }^{63}$ (1886-1960) konnte später aus den MolluscumKörperchen durch Trypsinverdauung tatsächlich Viruspartikel freisetzen und damit ihre Anwesenheit in den ZE nachweisen. Lépine ${ }^{64}$ gelang es durch elektronenoptische Beobachtungen zu bestätigen, dass bei Schafpocken die $\mathrm{ZE}$, in eine gelatineartige Matrix eingebettet, eine enorme Anzahl von $1 / 4 \mu$ grossen runden, ihrer Erscheinung nach mit Borrels Elementarkörpern identischen Partikeln enthielten.

Es blieb jedoch ungewiss, ob sämtliche Viren oder nur bestimmte Arten an ZE gebunden waren.

61 K. Lindner (Wien), «Zur Färbung der Prowazekschen Einschlüsse.»Cbl. Bakt. I. Abt. Orig. A (Jena), 55/5 (17. August 1910) 429-432.

62 Stanislaus von Prowazek (Hamburg), «Die Chlamydozoen als intracelluläre «symbiotische» Krankheitserreger.» Ergebn. wiss. Med. (Leipzig), 1/4 (Januar 1910) 135-145.

63 Ernest William Goodpasture/C. Eugene Woodruff (Nashville/Tenn.), «A Comparison of the Inclusion Bodies of Fowl-Pox and Molluscum Contagiosum.» Am. J. Path. (New York), 7/1 (January 1931) 1-7.

64 Pierre Lépine/Pascu Atanasiu/O. Croissant (Paris), «Aspect au microscope électronique des inclusions de la variole aviaire (Crops de Bollinger)». Ann Inst. Pasteur (Paris), 74 (1 Juillet 1948) 279-281. 


\section{Trennung der Viren von den Zelleinschlüssen}

Enrique Paschen ${ }^{65}$ (1860-1936) war es als erstem gelungen, das Variolavirus in der Pockenlymphe überzeugend darzustellen (Paschen-Körper). Er konnte jedoch nicht entscheiden, ob die Guarnierikörperchen durch das Eindringen des Erregers in die Zelle zustande kamen und eine Kolonie von mit Reaktionsstoffen der Zelle umhüllten Pockenviren verkörperten (Prowazeks Chlamydozoenlehre) oder die Reaktion auf ein lösliches Toxin darstellten (Hückels Toxinhypothese). Im Gegensatz zu Borrels Auffassung, wonach die Viren innerhalb der Zelle entstanden und erst später in das extrazelluläre Umfeld entladen wurden, vertritt Paschen die Ansicht, dass die Pockenviren sich stets ausserhalb der Zelle aufhielten und die Bildung der Guarnierikörperchen von aussen her durch ihre Toxine bewirkten.

Benjamin Lipschütz ${ }^{66}$ (1872-1931) hatte sich Borrels Auffassung angeschlossen. Doch anhand von Nocards und Roux ${ }^{167}$ Befunden bei der Peripneumonie der Rinder und seiner eigenen Beobachtungen bei Molluscum contagiosum ${ }^{68}$ hatte er bald erkannt, dass die Viren die Zelle nicht unbedingt zur Bildung einer Hülle bzw. eines Einschlusses reizten, sondern durchaus auch in ZE-freien Geweben vorkommen konnten. Er schloss hieraus, dass die winzigen Viren wichtiger sind als die Zellreaktion, welche auf ihr Eindringen unter Umständen stattfindet, und dass auch jene übertragbaren Krankheiten, bei denen die markanten ZE fehlten, gelegentlich durch Viren verursacht werden können.

\section{Affinität der Viren zu bestimmten Zellarten}

Lipschüt $^{69}$ hielt die mikroskopisch sichtbaren filtrierbaren Virusarten für obligate Zellparasiten, die den wichtigsten Teil ihres Lebenszyklus innerhalb und auf Kosten der Zelle verbringen und vollständig unfähig sind, sich aus-

65 Enrique Paschen (Hamburg), «Über den Erreger der Variolavaccine. Immunitätsverhältnisse bei Variolavaccine.» In: Rudolf Kraus/Constantin Levaditi (Hgg.) Handbuch der Technik und Methodik der Immunitätsforschung, Gustav Fischer Vlg., Jena (1911), Suppl 1, 465-517.

66 Benjamin Lipschütz (Wien), «Über mikroskopisch sichtbare, filtrierbare Virusarten. Über Strongyloplasmen.» Cbl. Bakt. Abt. I. Orig. A (Jena), 48/1 (Januar 1908) 77-90.

67 Nocard/Roux (oben Anm. 49).

68 Benjamin Lipschütz (Wien) «Untersuchungen über Molluscum contagiosum.» Dermat. Zschr. (Berlin), 14 (1908) 481-490.

69 Benjamin Lipschütz (Wien), «Über Chlamydozoa-Strongyloplasmen. II. Ueber den Bau und die Entstehung der 〈Zelleinschlüsse`.»Wien. klin. Wschr. 32/47 (19. November 1919) $1127-1131$. 
serhalb der Zelle zu vermehren. Die ZE-Bildung fasste Lipschüt ${ }^{70}$ hingegen als zellbiologischen Prozess auf, der nur in Zellen der aus dem äusseren Keimblatt hervorgegangenen Geweben (Hautepithel, Kornea, Gehirn) stattfand, zu welchen die Viren eine ausgeprägte Affinität zeigten. Diese später von Constantin Levaditi ${ }^{71}$ (1874-1928) weiterentwickelte Theorie war von der Beschränkung der ZE auf die genannten Gewebe veranschaulicht.

Als aber Albert Jesionek ${ }^{72}$ (Abb. 1, unten links) 1904 in den Geweben eines totgeborenen luetischen Fötus auffällige Kernkörper entdeckt hatte ${ }^{73}$ (Abb. 1, unten rechts) und später Tyzzer ${ }^{74}$ bei Varizellen, Torres ${ }^{75}$ bei Gelbfieber, Lipschütz ${ }^{76}$ bei Herpes und Zoster und weitere Forscher ${ }^{77}$ auch bei anderen pathologischen Zuständen vom Vorkommen von Kernkörpern berichteten, erkannte Lipschütz ${ }^{78}$, dass die Affinität der Erreger für bestimmte Zellarten eine Abstufung erfährt, indem das Virus sich entweder im Zytoplasma oder im Kern (selten in beiden) ansiedelt und ausschliesslich dort die Bildung von ZE auslöst. Er ordnete deshalb die Viren nach diesem Kriterium in drei Gruppen, die Karyo-, Zyto- und Karyozytooikongruppe, ein.

\section{Einschlusskrankheiten}

Der Nachweis von ZE konnte demnach unter Berücksichtigung ihres Sitzes und ihrer Beschaffenheit auch zu diagnostischen Zwecken verwendet

70 Benjamin Lipschütz (Wien), «Über Dermotropismus, Theorie der Pathogenese einiger menschlichen Dermatosen.» In: Stanislaus von Prowazek (Hrsg.) Handbuch der pathogenen Protozoen, Johann Ambrosius Barth Vlg., Leipzig, 1912, Bd. I, 243-246.

71 Constantin Levaditi (Paris), «Etude expérimentale de l'encéphalite dite «létargique». XVII. Comparaisons entre les divers virus neurotropes. Ectodermoses neurotropes.» Ann. Inst. Pasteur (Paris), 36 (1922) 144-151.

72 Albert Jesionek/Basilius Kiolemenoglou (München), «Über einen Befund von protozoënartigen Gebilden in den Organen eines hereditär-luetischen Fötus.» Münch. med. Wschr., 51/43 (25. Oktober 1904) 1905-1907.

73 Peter Diosi (Köln), «Albert Jesionek auf der Spur der Zytomegalie-Krankheit.» Gesnerus (Basel), 54/1-2 (Juni 1997) 96-112.

74 Edward Ernest Tyzzer (Boston), «The Histology of the Skin Lesions in Varicella.» J. med. Res. (Boston), 14 (1905) 361-392.

75 C. Magarinos Torres (Rio de Janeiro), «Inclusions nucléaires acidophiles (dégénérescence oxychromatique) dans le foie de Macacus rhesus inoculé avec le virus brésilien de la fièvre jaune.» C. R. Soc. Biol. (Paris), 99 (25 juillet 1928) 1344-1945.

76 Benjamin Lipschütz (Wien), «Untersuchungen über die Ätiologie der Krankheiten der Herpesgruppe (Herpes zoster, Herpes genitalis, Herpes febrilis).» Arch. Dermat. Syph. (Berlin), 136/3 (2. Dezember 1921), 428-482, hier 432.

77 Thomas M. Rivers/William S. Tillet (New York), «The Lesions in Rabbits Experimentally Infected by a Virus Encountered in the Attempted Transmission of Varicella.» J. exp. Med. (New York), 40/3 (September 3, 1924) 281-287.

78 Benjamin Lipschütz (Wien), «Der Zellkern als Virusträger (Die Karyooikongruppe der Chlamydozoa-strongylospasmen).» Cbl. Bakt. I. Abt. Orig. A (Jena), 87/4 (5. Dezember 1921) 303-310. 
werden. Lipschütz ${ }^{79}$ war sogar zu der Überzeugung gelangt, dass die ätiologische Diagnose der ansteckenden Hautkrankheiten eine rein zytologische Angelegenheit sei.

Er fasste die von ZE begleiteten Krankheiten unter der Bezeichnung «Einschlusskrankheiten» zusammen und teilte sie der ZE-aufweisenden Gewebeart entsprechend in mehrere Gruppen auf. Das Molluscum contagiosum zählte er zu den Epitheliosen, die Variola bzw. Vakzine zu den Dermatosen, die herpetischen Erkrankungen zu den Neurodermatosen, usw.

\section{Autokatalytische Nukleoproteine}

Die Morphologie war inzwischen in ihren Möglichkeiten, die Ursachen und die Natur von Krankheiten aufzuklären, allmählich hinter der sich rasch entwickelnden Biochemie und Physik zurückgeblieben. Das Interesse verlagerte sich daher von der visuellen Erscheinung der Gebilde auf ihre chemische Komposition. Durch diesen radikalen Wandel erfuhr auch die Virusforschung eine plötzliche Wende.

Edmund Cowdry ${ }^{80}(1888-1975)$ sah in den unsichtbaren Viren keine Mikroorganismen mehr, sondern Nukleoproteine bzw. autokatalytische Fermente und betrachtete deren Fortpflanzung als eine besondere Form der Proteinsynthese. Er brachte die Bildung unterschiedlicher Kernkörper auf den gemeinsamen Nenner einer Störung des zellulären Eiweissstoffwechsels, welcher neben den Viren auch durch andere mitosehemmende Einflüsse, wie sie von Blei ${ }^{81,82}$, Eisenoxid ${ }^{83}$, Bismut ${ }^{84}$

79 Benjamin Lipschütz (Wien), «Die Einschlußkrankheiten der Haut». In: Josef Jadassohn (Hrsg.) Handbuch der Haut- und Geschlechtskrankheiten, Julius Springer Vlg., Berlin, 1932, Bd.II.

80 Edmund Vincent Cowdry (St. Louis/Mo.), «The Microchemistry of Nuclear Inclusions in Virus Diseases.» Science (Washington), 68/1750 (July 13,1928) 40-41.

81 Stefan S. Nicolau/O. Baffet (Paris), «Formations simulant les inclusions à ultravirus, dans le rein et dans le foie d'animaux soumis à l'intoxication saturnienne.» C. R. Soc. Biol. (Paris), 126 (20. Novembre 1937) 659-663.

82 M. Wachstein (Brooklyn/N. Y.), «Acid-Fast Intranuclear Inclusion Bodies in Lead Poisoning. (Abstract)». Am. J. Path. (New York), 25 (1949) 608.

83 Peter K. Olitsky/Carl G. Harford (New York), «Intranuclear Inclusion Bodies in the Tissue Reactions Produced by Injections of Certain Foreign Substances.» Am. J. Path. (Boston), 13/5 (September, 1937) 729-747, sowie «Further Observations on Intranuclear Inclusions Produced by Non-Virus Materials.» Proc. Soc. exp. Biol. Med. (New York), 14/1 (February, 1938) 92-94.

84 Alfred Luger/Ernst von Lauda (Wien), «Über oxychromatische Veränderungen am Zellkern (Auf Grund von Untersuchungen von Herpes simplex, Zoster, Varizellen, Variola und Karpfenpocken). Ein Beitrag zur Kenntnis und Wertung einschlussartiger Gebilde.» Med. Klin. (Wien), 22/12 (19. März 1926) 456-458 und 22/13 (26. März 1926) 495-497. 
usw. ausgelöst werden und gelegentlich auch bei Gesunden vorkommen können ${ }^{85}$.

\section{Auflösung des Rätsels}

Nachdem Oswald Avery ${ }^{86}$ (1877-1955) 1944 die Nukleinsäuren als Träger der Erbanlage erkannt hatte und diese sich hinterher als Schlüsselsubstanzen der Eiweissbiosynthese herausstellten, gelang es den Biochemikern ${ }^{87}$, die virusbedingten Kernkörper anhand deren Empfindlichkeit auf DNase bzw. des positiven Ausfalls der von Robert J. W. Feulgen (1884-1955) entwickelten Nuklealfärbung klar von Kernkörpern anderer Natur zu unterscheiden.

Der Vergleich der von ein und demselben Virus in verschiedenen Zellarten bzw. von verschiedenen Viren in derselben Zellart ausgelösten ZE liess Goodpasture ${ }^{88}$ schliesslich erkennen, dass diese Gebilde keine Zellprodukte im Sinne Prowazeks oder Störungen des zellulären Eiweissstoffwechsels im Sinne Cowdrys, sondern Erzeugnisse der Viren, und zwar ausschliesslich jener Viren waren, welche die Wirtszelle langsam zerstörten.

Doch die Morphologie hatte inzwischen viel an Aussagekraft verloren. Nach Ausschöpfen der in der Anlage des Lichtmikroskops vorhandenen Möglichkeiten fehlten ihr nunmehr die zur Klärung der Einzelheiten nötigen hochentwickelten Methoden ${ }^{89}$.

\section{Adventus der Virologie}

1949 haben John Enders (1897-1985), Thomas Weller (geb. 1915) und Frederick Robbins ${ }^{90}$ (geb. 1916) mit der Isolierung des Poliovirus auf Einschichtzellkulturen eine atemberaubende Entwicklung der Virologie - von

85 Erich Schiller (Leipzig), «Über die Bedeutung von Kerneinschlüssen.» Dtsch. med. Wschr. (Stuttgart), 73/43-44 (19. November 1948) 573-574.

86 Oswald Theodore Avery/Colin M. MacLeod/Lyn McCarty (New York), «Studies on the Chemical Nature of the Substance Inducing Transformation of Pneumococcus Types.» J. exp. Med. (New York), 79 (1944) 137-158.

87 Jacob Dyckman (New York)/Joseph Bellamy (Hudson/N.Y.), «Histochemical Studies in Cytomegalic Inclusion Disease.» Arch. Path. (Chicago), 56/4 (October, 1953) 360-363.

88 Ernest W. Goodpasture (Nashville/Tenn.), «Intracellular Parasitism and the Cytotropism of Viruses.» South. med. J. (Birmingham, Ala.), 29 (1936) 297-302.

89 Margaret G. Smith (St. Louis/Missouri), «A Pathologists Approach to Viral Diseases.» Proc. Inst. Med. (Chicago), 25 (1964) 41-48.

90 John Franklin Enders/Thomas Huckle Weller/Frederick Chapman Robbins (Boston), «Cultivation of the Lansing strain of Poliomyelitis Virus in Cultures of Various Human Embryonic Tissues.» Science (Washington), 109/2 (February 11, 1949) 85-87. 
den anekdotischen Berichten über ZE bis hin zur Entzifferung der molekularen Struktur der Viren - eingeleitet.

$\mathrm{Da}$ die Geschichte hier allmählich in die Gegenwart mündet, genüge es zu erwähnen, dass die Brisanz dieser rasanten Entwicklung alle früheren Errungenschaften plötzlich als veraltet und überholt erscheinen liess und dass im Zuge des Wandels selbst die feinsinnigsten Erkenntnisse der Pioniere zu Selbstverständlichkeiten gerieten.

Die anfangs als Blastomyzeten oder Protozoen bzw. als Degenerationsprodukte der Zelle ausgelegten ZE haben sich dabei als grobe Erscheinungsbilder des intimen Prozesses der Virusproliferation herausgestellt. 